\title{
AXIOM: Advanced X-ray Imaging Of the Magnetosheath
}

S. Sembay ${ }^{1, \star}$, G. Branduardi-Raymont ${ }^{2}$, J. P. Eastwood ${ }^{3}$, D. G. Sibeck ${ }^{4}$, A. Abbey ${ }^{1}$, P. Brown ${ }^{3}$, J. A. Carter $^{1}$, C. M. Carr ${ }^{3}$, C. Forsyth ${ }^{2}$, D. Kataria ${ }^{2}$, S. Kemble ${ }^{5}$, S. Milan ${ }^{1}$, C. J. Owen ${ }^{2}$, A. M. Read ${ }^{1}$, L. Peacocke ${ }^{5}$, C. S. Arridge ${ }^{2}$, A. J. Coates ${ }^{2}$, M. R. Collier ${ }^{4}$, S. W. H. Cowley ${ }^{1}$, A. N. Fazakerley ${ }^{2}$, G. Fraser $^{1}$, G.H. Jones ${ }^{2}$, R. Lallement ${ }^{6}$, M. Lester ${ }^{1}$, F. S. Porter ${ }^{4}$, and T. Yeoman ${ }^{1}$

${ }^{1}$ Department of Physics and Astronomy, University of Leicester, Leicester, UK

2 Mullard Space Science Laboratory, University College London, Holmbury St Mary, Dorking, Surrey, UK

${ }^{3}$ Blackett Laboratory, Imperial College London, London, UK

${ }^{4}$ NASA Goddard Space Flight Centre, Greenbelt, MD, USA

${ }^{5}$ Astrium Ltd, Stevenage, UK

${ }^{6}$ LAMTMOS/Institute Pierre Simon Laplace, Paris, France

Received 30 May 2005, accepted 11 Nov 2005

Published online later

Key words Editorial notes - instruction for authors

AXIOM (Advanced X-ray Imaging Of the Magnetosphere) is a concept mission which aims to explain how the Earth's magnetosphere responds to the changing impact of the solar wind using a unique method never attempted before; performing wide-field soft X-ray imaging and spectroscopy of the magnetosheath, magnetopause and bow shock at high spatial and temporal resolution. Global imaging of these regions is possible because of the solar wind charge exchange (SWCX) process which produces elevated soft X-ray emission from the interaction of high charge-state solar wind ions with primarily neutral hydrogen in the Earth's exosphere and near-interplanetary space.

(C) 2006 WILEY.VCH Veriag GmbH \& Co. KGaA, Weinheim

\section{Introduction}

Traditionally the plasma and magnetic field environment of the Earth has been studied using in-situ measurements. Whilst this provides local information about plasma behaviour, instabilities and dynamics, this methodology lacks the global view which is necessary to understand the overall behaviour of the plasma. This problem can either be addressed by increasing the number of in-situ measurements via a multi-spacecraft approach or by remote imaging of relevant plasma emission processes. The former was the motivation behind the current four-spacecraft European Space Agency (ESA) Cluster mission and the proposed ESA Mclass candidate Cross-Scale and JAXA/CSA SCOPE missions which would have employed a total of twelve spacecraft if launched concurrently.

Remote imaging provides an alternative solution. Imaging has been used to study the Earth's aurora, for example in $\mathrm{X}$-rays with the PIXIE instrument on the Polar mission, and the inner magnetosphere with radio, ultraviolet and neutralatom imaging instruments on IMAGE. Imaging on larger scales to encompass the whole magnetosphere is possible using a wide-field $\mathrm{X}$-ray imager with the sensitivity to detect the soft X-ray line emission that arises from solar wind charge exchange; the process whereby high charge-state solar wind heavy ions exchange an electron with neutral hy-

\footnotetext{
* Corresponding author: e-mail: sfs5@star.le.ac.uk
}

drogen within the Earth's exosphere and subsequently relax by emitting X-ray (or ultraviolet) photons (Collier et al. 2010). The SWCX X-ray emissivity is directly related to the solar wind density (as well as the ion composition, average velocity and target density) and therefore provides a diagnostic of the mass flow of the incoming plasma through the region.

Theoretical work by (Robertson \& Cravens 2003) and (Robertson et al.2006) laid the groundwork for the feasibility of using imaging in this way by predicting the expected $\mathrm{X}$-ray emissivity within the magnetosheath in two cases; an average strength solar wind and that typical of a strong coronal mass ejection. The results suggested that the enchanced signal from the region would easily be detectable with an appropriately placed X-ray telescope with modest sensitivity. Direct measurements of terrestrial SWCX using relatively narrow field-of-view (FOV) X-ray telescopes such as on XMM-Newton (e.g. Snowden et al. 2004; Carter et al. 2011) have confirmed this view.

AXIOM (Advanced X-ray Imaging Of the Magnetosphere) is a mission concept that can provide this powerful new view of the dynamic plasma processes within the magnetosphere. The mission profile of AXIOM was developed for the ESA Medium Class Round 3 Announcement of Opportunity in 2011 and is described briefly in this paper. A more comprehensive description of AXIOM and its science goals is given in Branduardi-Raymont et al. (2011). 


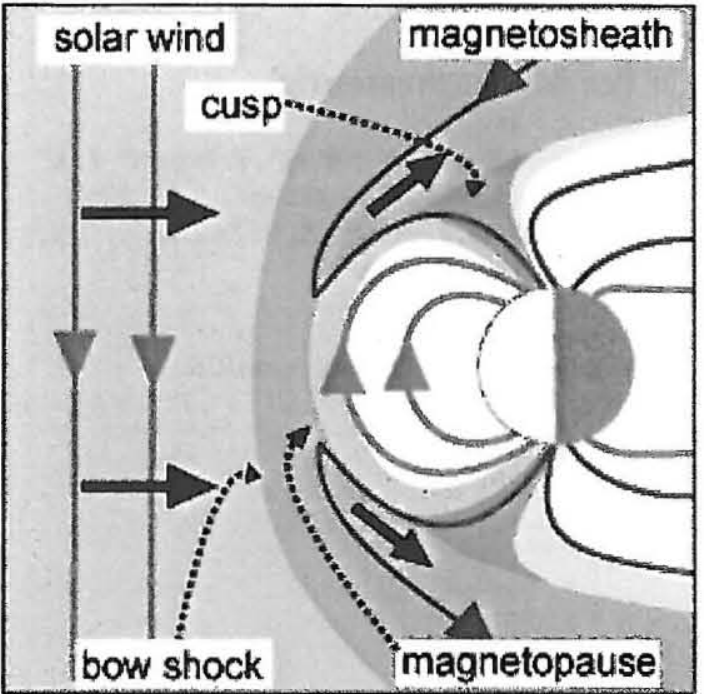

Fig.1 The dayside magnetosphere: The magnetopause represents the outer boundary of the magnetosphere, and is compressed on the dayside. Solar wind is processed at the bow shock, so that it may flow around the magnetopause.

Concepts with essentially the same primary science goals but designed around other opportunities are the LXO (Lunar X-ray Observatory) (Porter et al. 2008; Collier et al. 2008) and the STORM instrument (Kuntz et al. 2008; Collier et al. this proceedings) which was proposed as one instrument on the MAGIC (Magnetospheric Activation \& Geospace Ion Circulation) mission concept.

\section{Scientific Objectives}

The Earth's magnetic field carves out a cavity known as the magnetosphere in the collisionless supersonic and superAlfvénic solar wind plasma. The solar wind flow compresses the Sunward side of the magnetosphere but drags the nightside out into a long magnetotail. A sharp transition from tenuous magnetospheric plasmas to dense, shocked, solar wind plasmas marks the magnetopuase, the outermost boundary of the magnetosphere. Cusps at high latitudes on the dayside magnetopause denote locations where field lines divide to close either in the opposite hemisphere or far down the magnetotail. Weak field strengths within the cusps provide an opportunity for solar wind plasma to penetrate deep into the magnetosphere, all the way to the ionosphere. This scenario is illustrated in Fig. 1.

Because the solar wind is supersonic, a collisionless bow shock forms upstream of the magnetopause. The manner in which the solar wind is slowed, compressed, heated and diverted at planetary bow shocks is of key importance in understanding how the solar wind interacts with planetary magnetospheres, since it is the shocked magnetosheath plasma, not the pristine solar wind, that interacts with the magnetosphere at the magnetopause.

The position and shape of the magnetopause change continually as pressures within the Earth's magnetosphere attempt to respond to constantly varying solar wind dynamic pressures. The magnetosheath plasma flow depends on the shape and location of the shock, which in turn depends on the shape and location of the magnetopause, making an analytic solution intractable. The solar windmagnetosphere interaction on a global scale provides the primary science questions that AXIOM will address. These can be summarised as;

- Magnetopause physics

- How do upstream conditions control magnetopause position and shape and magnetosheath thickness?

- How does the location of the magnetopause change in response to prolonged periods of sub-solar reconnection?

- Under what conditions do transient boundary layers, such as the plasma depletion layer, arise?

- Cusp physics

- What are the size and shape of the cusps?

- How do the cusps move in response to changes in the solar wind?

- How does cusp density depend on magnetospheric coupling?

- Shock physics

- What controls the upstream bow shock location of a planetary magnetosphere?

- How does the steady-state thickness of a collisionless shock depend on the upstream conditions?

- Interaction of a Coronal Mass Ejection (CME) with the magnetosphere

\section{Mission Profile}

In order to obtain a global view of the Earth's magnetosphere we require a vantage point far out from the planet at a distance from the Earth of the order of tens of Earth Radii $\left(\mathrm{R}_{E}\right)$. Several orbit possibilities, both equatorial (circular) and polar (elliptical), were considered for AXIOM. Equatorial orbits provide the classical view from the side of the magnetosphere for a large fraction of the time, but suffer from having the (far too X-ray bright) Earth in the FOV for a significant fraction of the orbit. Polar elliptical orbits are much better at avoiding viewing the Earth, but imply regular passages through the Earth's radiation belts (unless perigee is above $\sim 6 \mathrm{R}_{E}$ ), which impose more severe radiation shielding requirements. In both configurations, we require that the Sun angle be above $45^{\circ}$ from the edge of the FOV, to help avoid stray-light being passed by the X-ray optic onto the detector.

After a mission analysis carried out by Astrium of the various possibilities we proposed that the AXIOM spacecraft be launched into a Lissajous orbit at the Earth-Moon 


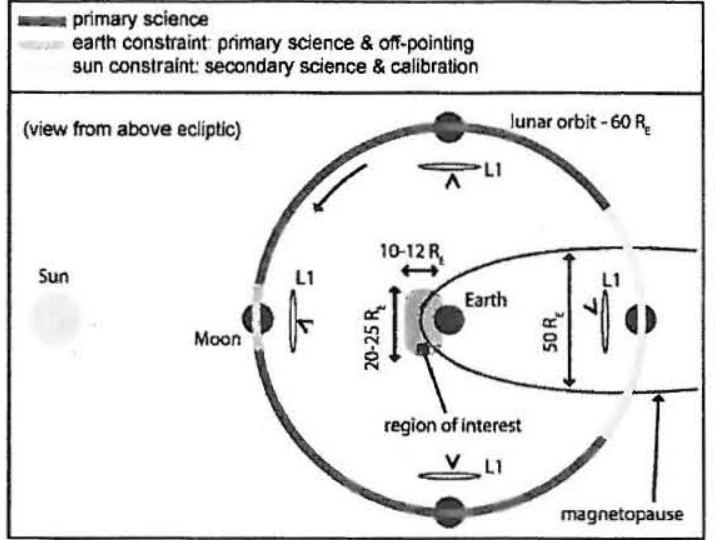

Fig.2 AXIOM orbit around the Earth-Moon L1 point (not to scale). The Lissajous orbit is represented by the narrow ellipses.

$\mathrm{L} 1$ point at a distance of $50-55 \mathrm{R}_{E}$ (Figure 2). This is a particularly mass-efficient orbit to reach (using a low speed approach to the Moon to enable a 'weak capture'). It was calculated that the ESA Vega launcher using a LISA Pathfinder Propulsion Module as the spacecraft bus could deliver into that orbit a payload of $451 \mathrm{~kg}$. The Lissajous orbit itself has a period of about 14 days (the larger L1 orbit being 28 days) and is somewhat unstable requiring some station keeping approximately every 7 days or so. The observing efficiency for the primary science target from this vantage point point was calculated to be around $58 \%$.

\section{Payload}

The primary science target of AXIOM is an extended structure around the Earth of size several $(\sim 10-20) \mathrm{R}_{E}$ emitting $\mathrm{X}$-rays at characteristic line energies in the range 0.1 to $2.5 \mathrm{keV}$. From the Earth - Moon L1 position this structure, encompassing the bow shock, magnetopause and magnetospheric cusps, has a scale size of several degrees. The prime instrument on AXIOM is a Wide-Field X-ray Imager (WFI) whose key requirements are defined to be;

1. Sensitivity to $\mathrm{X}$-rays in the energy range 0.1 to $2.5 \mathrm{keV}$ (primary science target), and to higher energies for calibration purposes

2. An energy resolution of $<65 \mathrm{eV}$ (FWHM) at $0.6 \mathrm{keV}$ sufficient to isolate the major $\mathrm{X}$-ray emission lines (primarily from OVII and OVIII in the energy band 0.5 to $0.7 \mathrm{keV}$ )

3. Imaging capability with an angular resolution of $\sim 7$ arcmin (equivalent to a scale size of $0.1 \mathrm{R}_{E}$ at a distance of $51 \mathrm{R}_{E}$ )

4. A wide FOV (baseline of $10^{\circ} \times 15^{\circ}$ )

5. Time resolution of $\sim 1$ minute.

Conventional Wolter-type X-ray optics of the nested shell design have at most a $\sim 1^{\circ} \mathrm{FOV}$, far too narrow to provide the wide-field capability required for AXIOM without incurring a prohibitive mass and cost penalty. The optic for the WFI therefore uses an ultra-lightweight micropore optic (MPO) array in a Lobster-Eye geometry (Brunton et al. 1997; Fraser et al. 2002). The basic optic technology is essentially identical to that described in Collier et al. (this proceedings) for the STORM prototype. Our baseline design has a focal length of $70 \mathrm{~cm}$. With the specified FOV this leads to an optic size of about $24 \mathrm{~cm} \times 36 \mathrm{~cm}$ and is formed from a tile of $6 \times 9=54 \mathrm{MPO}$ plates (of size $4 \mathrm{~cm} \times 4 \mathrm{~cm}$ ). The mass of the entire optic plane for this instrument is $<1$ $\mathrm{kg}$.

Imaging and spectroscopic capability in the detector is provided by wide-area X-ray sensitive CCDs. Our baseline device is a commercially available $4 \mathrm{k} \times 4 \mathrm{k}$ pixel CCD of physical size $6.14 \mathrm{~cm} \times 6.14 \mathrm{~cm}$. The detector plane must be half the size of optic to cover the FOV, hence the detector has 6 of these devices tiled in a $2 \times 3$ array. Table 1 summarises the key characteristics of the WFI.

In addition to the WFI, AXIOM also carries a plasma analysis package and a magnetometer. The plasma package consists of a Proton-Alpha Sensor (PAS) and an Ion Composition Analyser (ICA) which will derive basic solar wind parameters (e.g. density, velocity and temperature). The instruments are based on the Solar Orbiter Solar Wind Analyser (SWA) package (see ESA/SRE-2009-5, Yellow Book), optimised to address the AXIOM scientific requirements, which are overall less severe than those of the SWA. The magnetometer (MAG) measures the orientation and magnitude of the interplanetary magnetic field. The baseline design is a dual redundant digital fluxgate magnetometer consisting of two tri-axial fluxgate sensors. The fluxgate sensor also has a high technology readiness level having successfully flown on Cassini and Double Star.

The plasma package and magnetometer together can detect interplanetary shocks and solar wind discontinuities passing over the spacecraft. These interfaces, moving at hundreds of $\mathrm{km} \mathrm{s}^{-1}$ in the solar wind, take only a few seconds to pass over the spacecraft.

\section{Performance Assessment}

Here we briefly describe the performance assessement of the WFI, the prime instrument on AXIOM. Further details on the PAS, ICA and MAG instruments can be found in (Branduardi-Raymont et al. 2011).

The performance assessment of the WFI was based on the simulations of the expected X-ray emissivity of the magnetosheath and surrounding volume by Robertson et al. $(2003,2006)$. These represent two states of the solar wind; a quiescent solar wind (density $7 \mathrm{~cm}^{-3}$ and speed $400 \mathrm{~km} \mathrm{~s}^{-1}$ ) and a state representative of a storm (CME) which occurred in March 2001. The simulations predict peak X-ray emissivities of 8.8 and $160 \mathrm{keV} \mathrm{cm}^{-2} \mathrm{~s}^{-1} \mathrm{sr}^{-1}$ respectively (note the quiescent state model has no simulation of the cusp structure). 


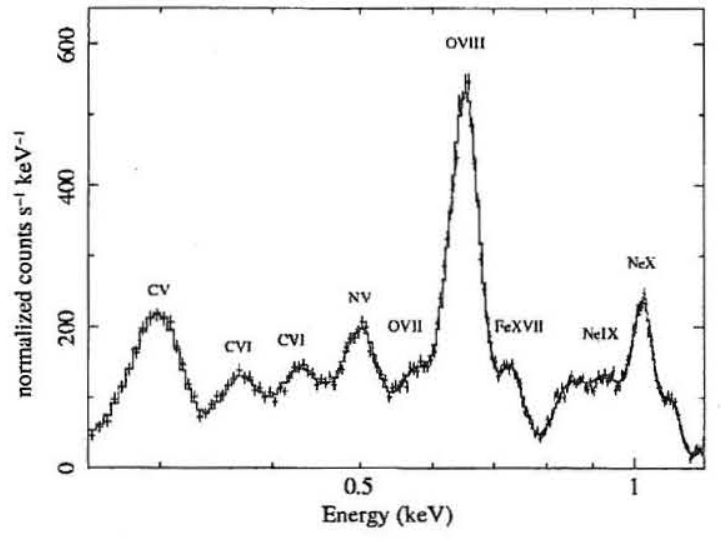

Fig.5 Simulated background-subtracted spectrum of SWCX emission from a CME assuming an exposure of $1 \mathrm{ks}$.

Figure 3 and Figure 4 (left two panels) show predicted WFI images derived from the SWCX emissivity maps folded through the detector response (assuming a viewing distance of $51 \mathrm{R}_{E}$ from the Earth) with an expected background component added. The integration times are $100 \mathrm{~s}$ and $1 \mathrm{ks}$ for the storm simulation and $1 \mathrm{ks}$ and $10 \mathrm{ks}$ for the quiescent state simulation. The scale of these images is counts $\mathrm{ks}^{-1}$ per $0.1^{\circ}$ pixel. Each image has been smoothed to bring out detail. We also show (right panels) the significance of the SWCX emission in number of sigmas above the estimated background. The significance maps are binned on a $0.5^{\circ}$ pixel scale. The background is a combination of the typical diffuse sky background, $\sim 8 \mathrm{keV} \mathrm{cm}^{-2} \mathrm{~s}^{-1} \mathrm{sr}^{-1}$ at $1 \mathrm{keV}$, and an estimated instrumental background of $\sim 10^{-4}$ cts $\mathrm{s}^{-1} \mathrm{keV}^{-1}$ per square arcmin at $1 \mathrm{keV}$, both derived from XMM-Newton data.

In the storm simulation the global structure of the magnetosheath is easily visible above the background in only $100 \mathrm{~s}$. The position and extent of the cusps, for example, should be detectable to within a fraction of an $R_{E}$ on timescales of a few minutes during periods of high solar activity. During average solar wind conditions we would require integration times of around $1 \mathrm{ks}$.

In addition the WFI will also be able to accumulate high precision SWCX spectra. The moderate energy resolution of the CCD detectors will enable the principal lines from the major ions to be resolved and solar wind abundances can be derived directly from the $\mathrm{X}$-ray data. Although the effective area of the WFI is modest the wide FOV gives the instrument an enormous grasp (defined as effective area times the FOV area) for collecting photons from diffuse emission, 25 times that of XMM-Newton at $0.6 \mathrm{keV}$. Figure 5 shows a simulated background-subtracted spectrum of SWCX emission from a CME assuming an exposure of $1 \mathrm{ks}$. The spectral model was directly based on a SWCX spectrum from a CME as observed by XMM-Newton (Carter et al. 2010).

\section{Summary}

The concept of using X-ray emission from SWCX interactions to image the dynamic interaction between the solar wind and the magnetosphere has the potential to revolutionise this field of study. A better understanding of the energy transfer from the solar wind into the Earth environment is of crucial importance in our modelling and forecasting of space weather effects. The AXIOM concept as described here is relatively low cost, calculated to be about half the cost of a typical ESA M class mission, using technology which is at a high technological readiness level. AXIOM directly addresses the question raised in ESA's Cosmic Vision quest, 'How does the Solar System work?'.

Acknowledgements. The authors would like to thank Astrium (www.astrium.eads.net) for their support in developing the AXIOM spacecraft and mission profile, E2V (www.e2v.com) for their technical assistance with the design of the WFI detector plane and Photonis (www.photonis.com) for their long collaboration with Leicester University in the field of micropore optics.

\section{References}

Branduardi-Raymont, G., Sembay, S. F., Eastwood, J. P., et al. 2011, Experimental Astronomy, 102

Brunton, A. N., Fraser, G. W., Lees, J. E., \& Turcu, I. C. E. 1997, Appl. Opt., 36, 5461

Carter, J. A., Sembay, S., \& Read, A. M. 2010, MNRAS, 402, 867

Carter, J. A., Sembay, S., \& Read, A. M. 2011, A\&A, 527, A115+

Collier, M. R., Carter, J., Cravens, T., et al. 2008, LPI Contributions, 1415, 2082

Collier, M. R., Sibeck, D. G., Cravens, T. E., Robertson, I. P., \& Omidi, N. 2010, Eos Trans. AGU, 91, 213

Fraser, G. W., Brunton, A. N., Bannister, N. P., et al. 2002, in Society of Photo-Optical Instrumentation Engineers (SPIE) Conference Series, Vol. 4497, Society of Photo-Optical Instrumentation Engineers (SPIE) Conference Series, ed. K. A. Flanagan \& O. H. W. Siegmund, 115-126

Kuntz, K., Collier, M. R., Sibeck, D. G., et al. 2008, AGU Fall Meeting Abstracts, A1630+

Porter, F. S., Abbey, T. F., Bannister, N. P., et al. 2008, in Proceedings of the SPIE Conference Series, Vol. 7011

Robertson, I. P., Collier, M. R., Cravens, T. E., \& Fok, M.-C. 2006, Journal of Geophysical Research (Space Physics), 111, 12105

Robertson, I. P. \& Cravens, T. E. 2003, Geophys. Res. Lett., 30, 080000

Snowden, S. L., Collier, M. R., \& Kuntz, K. D. 2004, ApJ, 610 , 1182 

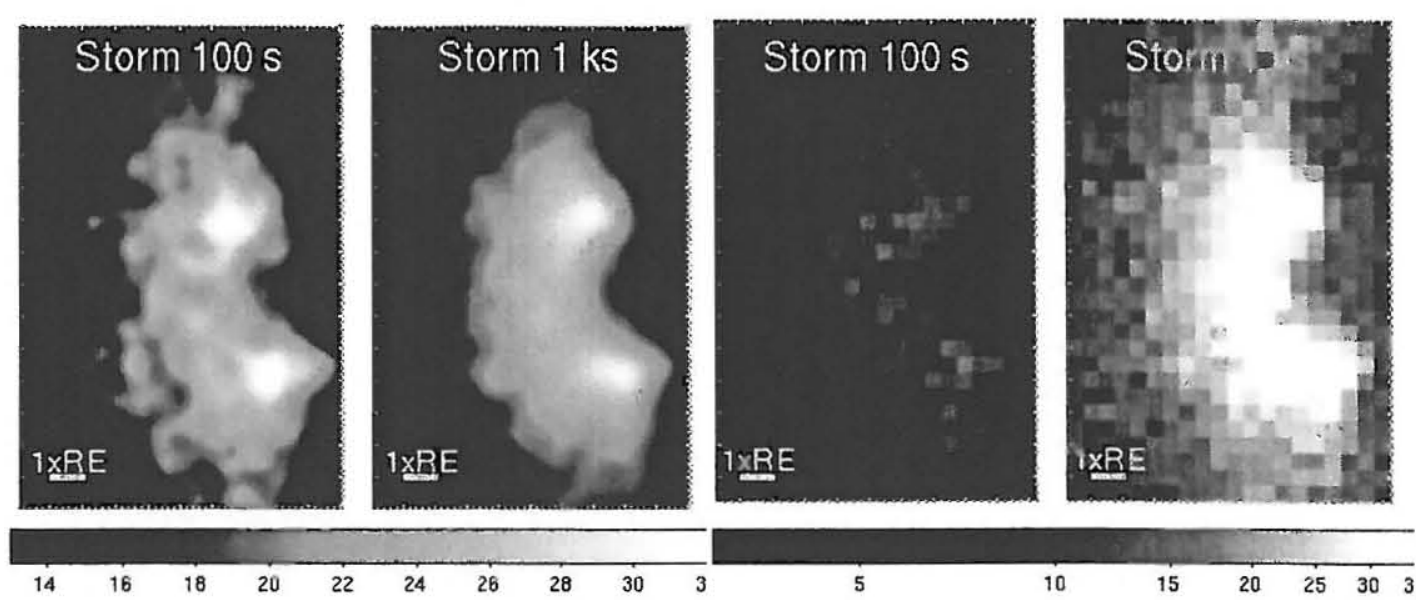

Fig.3 Simulated WFI images (left two panels) and significance maps (right two panels) for solar wind state represtative of a Coronal Mass Ejection given exposures of $100 \mathrm{~s}$ and $1 \mathrm{ks}$.
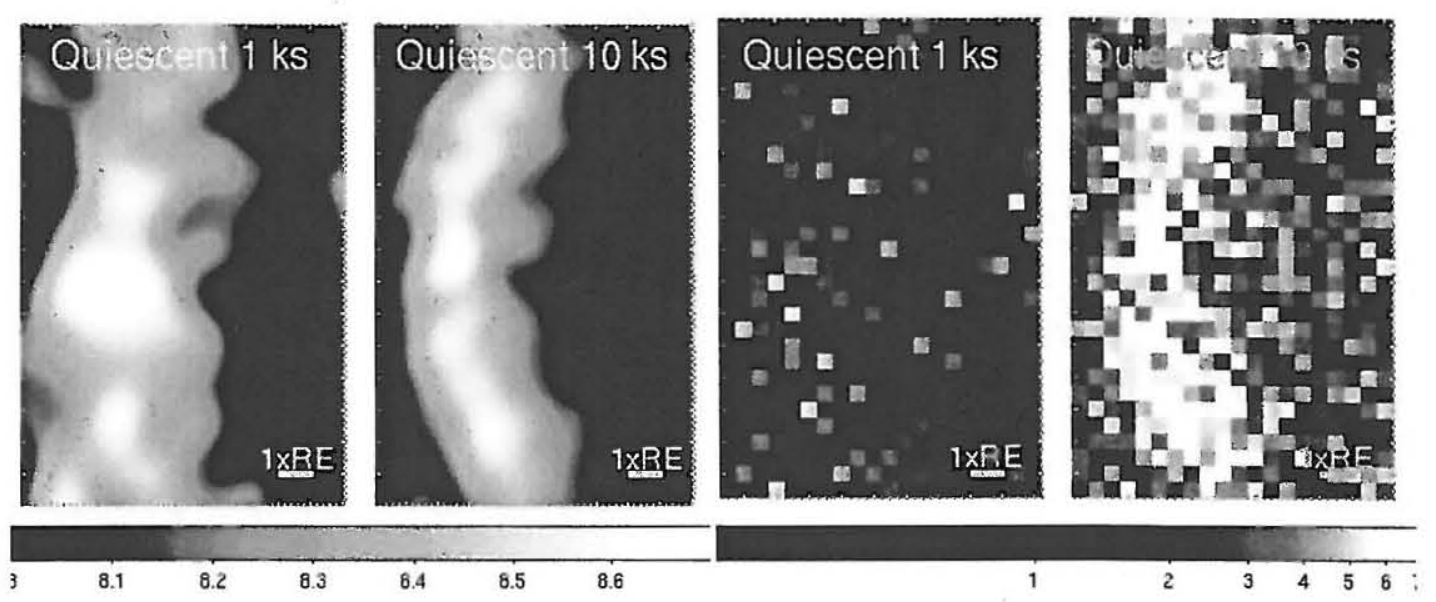

Fig.4 As Figure 3 but for a quiesecent solar wind and exposures of $1 \mathrm{ks}$ and $10 \mathrm{ks}$.

Table 1 Main WFI characteristics.

\begin{tabular}{|l|c|l|}
\hline Attribute & value & Notes \\
\hline Optic FOV & $10^{\circ} \times 15^{\circ}$ & \\
Optic angular resolution & 2 arcminute FWHM & \\
Optic focal length & $70 \mathrm{~cm}$ & \\
Optic effective area & $58 \mathrm{~cm}^{2}$ & \\
Total effective area at $0.6 \mathrm{keV}$ & $37 \mathrm{~cm}^{2}$ & Asuming CCD QE $\sim 0.8$ and $1600 \AA$ Polyimide $+800 \AA$ Al UV filter \\
Detector energy resolution at $0.6 \mathrm{keV}$ & $65 \mathrm{eV} \mathrm{FWHM}$ & Assuming $6 \mathrm{e}^{-}$readout noise \\
Detector pixel size & $60 \mu \mathrm{m}$ & Assuming onboard $4 \times 4$ binning of native $15 \mu \mathrm{m}$ pixels \\
Time resolution & 31.1 seconds & 30 s frame time $+1.1 \mathrm{~s}$ readout time \\
\hline
\end{tabular}


